\title{
32) EFFECTS OF AGING AND SODIUM-LOADING ON THE URINARY EXCRETION OF THE ENDOGENOUS DIGITALIS-LIKE SUBSTANCE IN SHR
}

Makoto Matsusawa, Hakuo Takahashi, Keisuke Suga, Iwao Ikegaki, Masato Nishimura, Manabu Yoshimura, and Masao Nakagawa. 2nd Department of Medicine, Kyoto Prefectural University of Medicine, Kyoto 602

Since essential and experimental hypertension are closely associated with excess intake of sodium, the substance related to sodium metabolism must be implicated in the pathogenesis of hypertension. It has been suggested that the endogenous digitalis-like substance (EDLS) has been increased with sodiumloading in animals and humans. Accumulated evidence suggests that the sympathetic outflow is increased in SHR. Because the EDLS is produced in the hypothalamus, and potentially elevates the peripheral sympathetic discharge (Takahashi et al, Jpn. Circ. J. 51:1199-1207, 1987), the circulating EDLS could be involved in the pathogenesis of spontaneous hypertension in rats. In the present study, we investigated the effects of aging and sodium-loading on the urinary excretions of the endogenous EDLS using SHR and WKY.

SHR and WKY were fed with a regular diet containing $0.8 \% \mathrm{NaCl}$. Rats aged 8 and 13 week-old, were measured the systolic blood pressure by a tail-cuff method. And they were housed in metabolic cages for one day with a regular diet and then the diet was switched to a high-sodium diet containing $8 \% \mathrm{NaCl}$ for another one day. The urine collected in a container with $1 \mathrm{~m} 1$ of $3 \mathrm{~N} \mathrm{HC} 1$ for 24 hours was used for the measurement of the EDLS. The EDLS was measured by the digoxin-radioimmunoassay (RIA), which are specific for the determination of the EDLS as described e1sewhere (Takahashi et a 1, J. Hypertension 7:s314, 1986)

The blood pressure in SHR was higher than that in WKY in both 8 and 13 weeks old. The urinary volume in SHR was significantly smaller than that in WKY at the 14 weeks old rats fed with a regular diet $(30 \pm 3 \mathrm{~m} 1$ v $5.24 \pm 4 \mathrm{~m} 1$, $p<0.01$ ), and as a whole the volume in SHR tended to be smaller than that in WKY. Urinary excretion of EDLS increased as they got older ( 8 vs 13 week-old SHR:2.3 $\pm 0.5 \mathrm{ng} / \mathrm{day}$ vs. $10.5 \pm 7.2 \mathrm{ng} / \mathrm{day}, \mathrm{p}<0.01$, WKY $1.9 \pm 0.8 \mathrm{ng} / \mathrm{day}$ vs. $9.4 \pm 3.7$ ng/day, $p<0.01$ ). Although sodium loading for 1 day increased the urinary output of the EDLS, the relative increase was smaller when the rats got older (SHR; $466 \%$ at 8 week-old, $164 \%$ at 13 week-old, WKY; $496 \%$ at 8 week-old and $242 \%$ at 13 week-old).

Although the underlying mechanism in increasing the urinary output of the EDLS with increasing age has not yet been clarified, this could be due to the deteriorated natriuretic activity in the older rats. In accord with this. plasma renin activity decreases with increasing of age in humans probably due to sodium retention. The reason why the EDLS responses to sodium-loading was attenuated despite the increased baseline level in the 13 week-old rats is uncertain. Since blood pressure increases even when SHR was fed with a lowsalt diet, the sodium intake may not be essentially involved in the spontaneous hypertension. Eventually, because the urinary output of the EDLS in SHR was not increased in SHR either with a regular or a high-salt diet in both age groups of 8 and 13 weeks in the present study, it is thought that the EDLS does not play an important role in the blood pressure regulation in SHR. 